2. Treasure T, Lang-Lazdunski L, Waller D, Bliss JM, Tan C, Entwisle J, et al. Extrapleural pneumonectomy versus no extra-pleural pneumonectomy for patients with malignant pleural mesothelioma: clinical outcomes of the mesothelioma and radical surgery (MARS) randomised feasibility study. Lancet Oncol. 2011;12: 763-72.

3. Sugarbaker DJ, Richards WG, Bueno R. Extrapleural pneumonectomy in the treatment of epithelioid malignant pleural mesothelioma: novel prognostic implications of combined N1 and N2 nodal involvement based on experience in 529 patients. Ann Surg. 2014;260:577-80; discussion 580-2.

4. Lim E, Darlison L, Edwards J, Elliott D, Fennell DA, Popat S, et al. Mesothelioma and radical surgery 2 (MARS 2): protocol for a multicentre randomised trial comparing (extended) pleurectomy decortication versus no (extended) pleurectomy decortication for patients with malignant pleural mesothelioma. BMJ Open. 2020;10:e038892.

5. Hasegawa S, Yokoi K, Okada M, Tanaka F, Shimokawa M, Daimon T, et al. Neoadjuvant pemetrexed plus cisplatin followed by pleurectomy for malignant pleural mesothelioma. J Thorac Cardiovasc Surg. 2022;163: 1940-7.e5.

6. Shintani Y, Hasegawa S, Takuwa T, Aoe K, Kato K, Fujimoto N, et al. Prospective registry database of patients with malignant mesothelioma: directions for a future Japanese registry-based lung cancer study. J Thorac Dis. 2018;10: 1968-71.
See Article page 1940.

\section{Commentary: Surgical standardization in mesothelioma trials}

\section{Valerie W. Rusch, MD, FACS}

In this issue of the Journal, Hasegawa and colleagues ${ }^{1}$ report the results of a small, multicenter, single-arm, Phase II clinical trial testing the use of neoadjuvant chemotherapy followed by surgical resection in selected patients with malignant pleural mesothelioma (MPM). In essence, this study corroborates the experience reported in earlier trials of similar design showing the feasibility of preoperative chemotherapy followed by surgical resection using extrapleural pneumonectomy (EPP), pleurectomy/decortication (P/D), or extended pleurectomy/decortication (EPD). ${ }^{2,3}$

Perhaps the most important aspect of this study is the rigor of the approach to surgical resection and to documentation of surgical outcomes. Once it became recognized that EPP was associated with a greater morbidity and mortality relative to $\mathrm{P} / \mathrm{D}$ or $\mathrm{EPD}$, with a potential survival benefit seen only in younger patients with node-negative epithelioid

From the Department of Surgery, Memorial Sloan Kettering Cancer Center, New York, NY; and American College of Surgeons, Chicago, Ill.

Dr Rusch's work is supported in part by the National Institutes of Health/National Cancer Institute Cancer Center Support Grant P30 CA008748.

Disclosures: Dr Rusch reported institutional clinical trial funding from Genentech and travel reimbursement from Intuitive Surgical for robotic mentoring.

The Journal policy requires editors and reviewers to disclose conflicts of interest and to decline handling or reviewing manuscripts for which they may have a conflict of interest. The editors and reviewers of this article have no conflicts of interest.

Received for publication Aug 27, 2021; revisions received Aug 27, 2021; accepted for publication Aug 31, 2021; available ahead of print Sept 30, 2021.

Address for reprints: Valerie W. Rusch, MD, FACS, Memorial Sloan-Kettering Cancer Center, 1275 York Ave, New York, NY 10065 (E-mail: ruschv@mskcc.org).

J Thorac Cardiovasc Surg 2022;163:1949-50 $0022-5223 / \$ 36.00$

Copyright (c) 2021 Published by Elsevier Inc. on behalf of The American Association for Thoracic Surgery

https://doi.org/10.1016/j.jtcvs.2021.08.077

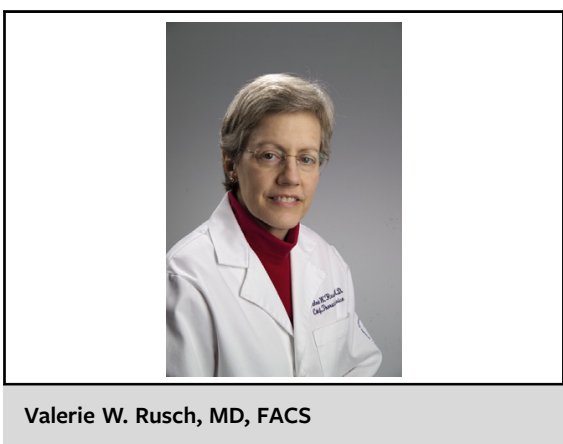

CENTRAL MESSAGE

This study establishes an important bar in terms of technical quality and surgical reporting in clinical trials.

tumors, ${ }^{4,5}$ the thoracic surgical community shifted to performing lung-sparing operations (P/D or EPD). Relative to EPP, these procedures are subject to greater variability in the type of resection because of the heterogeneity in tumor extent that occurs in MPM and a lack of standardization in surgical technique. Since the historical term P/D was too vague a description for lung-sparing operations, the IASLC (International Association for the Study of Lung Cancer) MPM Staging and Prognostic Factors Subcommittee proposed using the term EPD to describe parietal pleurectomy with or without visceral pleural removal (decortication) plus resection of the diaphragm and/or pericardium and retaining the term P/D to describe pleurectomy and decortication only. ${ }^{6}$ This nomenclature was widely adopted but remains insufficient to describe the variations in the extent of lung-sparing operations which in turn influence surgical risk and outcomes, particularly in the context of multimodality treatment. The IASLC MPM subcommittee, 
including some of the authors of this multicenter study, is currently developing a more granular nomenclature to describe the extent of resection, the potential extent of residual tumor at the end of the operation, and to propose a template for a standardized electronic operative report. Data elements being gathered by the IASLC MPM subcommittee in preparation for the Ninth edition of the international staging system already require extensive details regarding the details of the operation performed since these may influence long-term outcomes.

This study by Hasegawa and colleagues sets an important bar in surgical trial design in MPM. Detailed descriptions of the operation and educational sessions provided guidelines for participating surgeons, many of whom were relatively inexperienced in these long, complex, and potentially morbid procedures. Surgical videos were used to verify the extent of resection. Surgical details were carefully collected and an internationally accepted system for adverse event reporting was used to record postoperative complications. A reproducible surgical end point, MCR (macroscopic complete resection), was used as the primary end point. Consequently, although the trial was small, the results were high quality. The authors should be congratulated on the high standards established in this study.

\section{References}

1. Hasegawa H, Yokoi K, Okada M, Tanaka F, Shimokawa M, Daimon T, et al. Neoadjuvant pemetrexed plus cisplatin followed by pleurectomy for malignant pleural mesothelioma. J Thorac Cardiovasc Surg. 2022;163:1940-7.e5.

2. Krug LM, Pass HI, Rusch VW, Kindler HL, Sugarbaker DJ, Rosenzweig K, et al. Multicenter phase II trial of neoadjuvant pemetrexed plus cisplatin followed by extrapleural pneumonectomy and radiation for malignant pleural mesothelioma. $J$ Clin Oncol. 2009;27:3007-13.

3. Rimner A, Zauderer MG, Gomez DR, Adusumilli PS, Parhar PK, Wu AJ, et al. Phase II study of hemithoracic intensity-modulated pleural radiation therapy (IMPRINT) as part of lung-sparing multimodality therapy in patients with malignant pleural mesothelioma. J Clin Oncol. 2016;34:2761-8.

4. Rusch VW, Giroux D, Kennedy C, Ruffini E, Cangir AK, Rice D, et al. Initial analysis of the International Association for the Study of Lung Cancer Mesothelioma database. J Thorac Oncol. 2012;7:1631-9.

5. Flores RM, Pass HI, Seshan VE, Dycoco J, Zakowski M, Carbone M, et al. Extrapleural pneumonectomy (EPP) versus pleurectomy/decortication (P/D) in the surgical management of malignant pleural mesothelioma (MPM): results in 663 patients. J Thorac Cardiovasc Surg. 2008;135:620-6.

6. Rice D, Rusch V, Pass H, Asamura H, Nakano T, Edwards J, et al. Recommendations for uniform definitions of surgical techniques for malignant pleural mesothelioma: a consensus report of the International Association for the Study of Lung Cancer International Staging Committee and the International Mesothelioma Interest Group. J Thorac Oncol. 2011;6:1304-12. 